\title{
Hybrid spectrum access model using game theory approach for multi-channel heterogeneous mobile cognitive radio wireless sensor network
}

\author{
Saroja T.V ${ }^{1}$, Dr. Lata L. Ragha ${ }^{2}$, Prof. Satyendra Kumar Sharma ${ }^{3}$ \\ ${ }^{1}$ Faculty of Computer Engineering, Pacific Academy of Higher Education and Research University, India \\ ${ }^{2}$ Department of Computer Engineering Terna Engineering College Nerul, India \\ ${ }^{3}$ Modern Institute of Technology \& Research Centre Alwar (Raj), India
}

\begin{tabular}{l}
\hline \hline Article Info \\
\hline Article history: \\
Received Jan 22, 2019 \\
Revised Apr 19, 2019 \\
Accepted May 21, 2019 \\
\hline
\end{tabular}

\section{Keywords:}

Cognitive radio network

Game theory

MAC

Spectrum access

Wireless sensor network

\begin{abstract}
An efficient spectrum access and medium access control (MAC) design is required to enable wireless sensor network (WSN) with cognitive Internet of Things (IoT) that enables presence of sensor network with existing wireless infrastructure. Designing spectral, energy efficient and fair spectrum access design for IoT in CR-WSN is challenging especially for heterogeneous mobile CR-WSN. Existing model induces high collision and energy overhead. As a result, they are not efficient in utilizing spectrum. To overcome the research issues, this paper proposes a hybrid spectrum access model (HSAM) for multi-channel heterogeneous mobile CR-WSN. Next it elaborates on an accurate feasible channel accessible likelihood estimation. Further, we present a game theory model for HSAM for existence of finegrained Nash equilibrium. Experiments are conducted to evaluate the performance of HSAM-CRWSN over existing model. The outcome shows HSAM-CRWSN attains significant performance improvement over state-ofart technique in terms of energy efficiency, throughput, successful packet transmission and packet collision. The HSAM-CRWSN improves the overall spectrum efficiency of CR-WSN.
\end{abstract}

Copyright $@ 2019$ Institute of Advanced Engineering and Science. All rights reserved.

\section{Corresponding Author:}

Saroja T.V,

Faculty of Computer Engineering,

Pacific Academy of Higher Education and Research University,

Debari, Udaipur Rajasthan 313003, India.

Email: sarojatv2005@gmail.com

\section{INTRODUCTION}

A wireless sensor network is self-composed of low cost and tiny sensor devices which are deployed in hazardous location where recharging of sensor devices is almost impossible. The affordable price of sensor device has triggered various applications [1-2] such as Big Data, IoT etc. These applications requires large amount of data exchange (throughput), hierarchical structure, low latency service delivery with low energy dissipation and higher spectrum availability [3]. However, the $2.4 \mathrm{GHz}$ frequency channel is already crowded with various wireless application such as Bluetooth and Wi-Fi and Fifth generation network is expected to come which further requires efficient spectrum access design.

Cognitive radio [4] networks can be considered for addressing spectrum access for future cognitive network based IoT technology. Cognitive IoT (COIT) systems offers enhanced intelligence for achieving efficient data sensing and analysis [5] specifically for WSN. To fully incorporate COIT in WSN, smart and improved medium access control (MAC) design supporting the presence of sensor network with existing wireless infrastructure is required. Further, cognition in spectrum access need to be addressed to fully incorporate COIT. That is, most of spectrum is utilized on and off and they would be congested on certain 
sections of the spectrum. There are instances of artificial and real spectrum scarcity [6] that make it necessary for requirement of efficient use of spectrum. The architecture of cognitive radio wireless sensor network (CR-WSN) is shown in Figure 1.

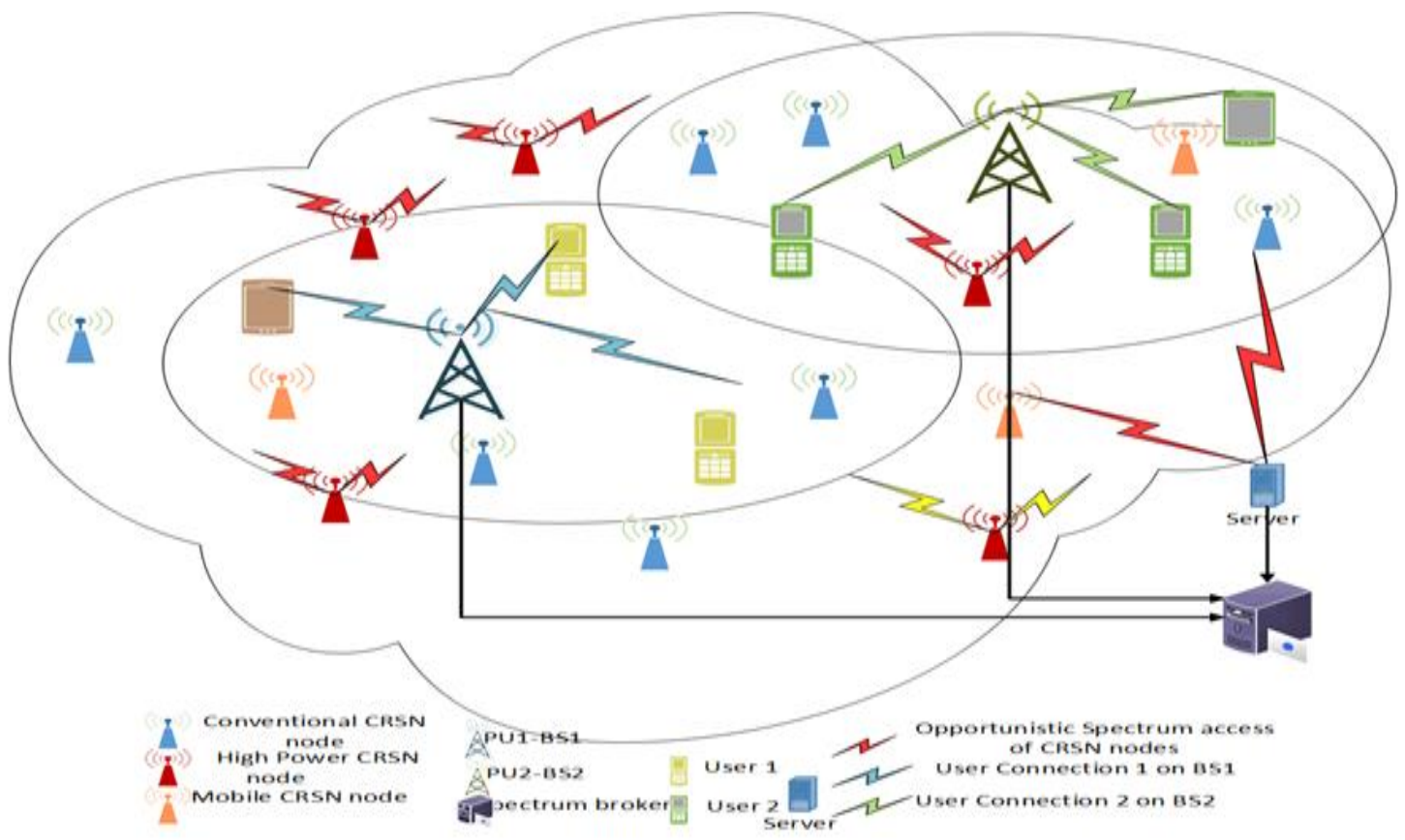

Figure 1. Architecture of Cognitive Radio Wireless Sensor Network

Spectrum inefficiency can be addressed by adopting opportunistic spectrum access (OSA) mechanism which is also referred to as dynamic spectrum access (DSA) mechanism. OSA is one of the main features of CR where wireless device can sense, learn and adapt. Though CRSN aid in improving IoT network performance and user requirements, sensor devices incur energy overhead to perform spectrum sensing and spectrum sharing [7] for achieving QoS and high throughput requirement [8-9]. These applications require distributed channel access considering unknown network information. Such design was presented for single cognitive user in [10] and for multi-user in [11]. However, considering multi-user scenario it induces collision in network. Because of interference and some user on CR-WSN are selfish. To reduce collision, priority [12], random access [13], and fair resource allocation [14] model has been presented. However, these models are limited to provide only one channel at a time to a cognitive user. As a result, if a selected channel is busy the user needs to wait another slot even if another channel is free at that instance of time. As a result, the spectrum is not utilized properly.

To overcome the research challenges in providing fair and efficient resource allocation. This paper presents Hybrid spectrum access model for distributed CR-WSN. For achieving fair resource allocation, game theory model is being adopted by various existing research [15-17]. However, these models are designed considering static secondary user, single channel, and homogenous environment and in [18] a game theory model is discussed considering multi-channel environment and mobile secondary device. However, the existence of Nash equilibrium is not explored which incurs channel switching overhead due to interference when mobility speed is increased. To address overhead due to mobility, this work presents an accurate estimation of channel accessibility likelihood using continuous-time Markov chain [19-20] and a Hybrid spectrum access model (HSAM) for multi-channel CR-WSN. Further, a game theory model for HSAM for existence of pure or fine-grained Nash equilibrium is presented.

The contribution of research is as follows:

a. This work presents a Hybrid spectrum access model (HSAM) for multi-channel heterogeneous mobile CR-WSN.

b. We discuss an efficient channel state estimation (i.e., feasible channel accessible likelihood) model for mobile CR-WSN.

c. Finally, we present a game theory model for HSAM for existence of fine-grained Nash equilibrium.

d. Reduce energy overhead of sensor device of mobile CR-WSN.

Hybrid spectrum access model using game theory approach for multi-channel heterogeneous... (Saroja T.V) 
e. HSAM attain good performance in terms of throughput, successful packet transmission and collision when compared to state-of-art technique.

The paper is organized as follow: The proposed hybrid spectrum access model for CR-WSN is presented in section two. In section three the simulation outcome and analysis is presented. In the last section, the paper is concluded with future research.

\section{HYBRID SPECTRUM ACCESS MODEL FOR COGNITIVE RADIO WIRELESS SENSOR NETWORK}

This work presents a hybrid spectrum access model for cognitive radio wireless sensor network (HSAM-CRWSN). Further, we present a fine-grained Nash equilibrium existence for Random MAC for distributed mobile cognitive radio wireless sensor network. In our previous work [19-20], we obtained model to define channel availability model, i.e., the feasible channel accessible of each channel.

\subsection{Feasible Channel Accessible Model} as follows:

The mean fraction channel accessibility of an area at any instance of channel $c$ represented by $\omega_{c}$ is

$$
\omega_{c}=\left(1-\delta_{c}\right)+\delta_{c} \alpha_{I, c}=1-\delta_{c} \alpha_{B, c}
$$

Where $\delta_{c}=4 T^{2} / A_{l, i}^{2}, \alpha_{I, c}$ are the steady mode likelihood that primary transmitter being active on channel $c$ and $\alpha_{B, c}$ are the steady mode likelihood indicating that primary transmitter is inactive on channel $c$. More detail of channel availability can be obtained in [1]. The state $M_{N, c}$ concludes when the sensor devices moves out of the coverage area of the primary transmitting user. Thus, $K_{N, c}$ is an exponential distribution with respect to parameter $\mu_{N, c}$, where,

$$
\mu_{N, c}=\mu_{b, c}+\frac{s^{\prime}}{f\left(A_{l, c}-T_{c}\right)} \alpha_{b, c}
$$

Here we consider an equilibrium model i.e.,

$$
\alpha_{X, c} \mu_{X, c}=\alpha_{N, c} \mu_{N, c}
$$

Where $\alpha_{X, c}=\omega_{c}$, and $\alpha_{N, c}=1-\omega_{c}$, therefore we can obtain the $M_{X, c} \rightarrow M_{N, c}$ transition rate $\mu_{X, c}$ as follows:

$$
\begin{aligned}
& \mu_{X, c}=\frac{\omega_{c}}{1-\omega_{c}} \mu_{N, c} \\
& =\frac{\delta_{c} \alpha_{b, c}}{1-\delta_{c} \alpha_{b, c}}\left(\mu_{b, c}+\frac{s^{\prime} \cdot \alpha_{b, c}}{f\left(A_{l, c}-T_{c}\right)}\right)
\end{aligned}
$$

Therefore, $K_{X, c} \sim \operatorname{Exp}\left(\mu_{X, c}\right)$. The feasible channel accessibility $\beta$ of channel $c$ as the mean time period in which channel $c$ is accessible for sensor device to communicate is computed using following:

$$
\beta_{c}=\varphi_{c} \cdot K_{X, c}^{\prime}=\frac{\varphi_{c}}{\mu_{X, c}}
$$

where $\varphi_{c} \in(0,1)$ is the factor of interference or the acceptance level of interference of the primary user . An important thing to be noted here is that a larger value of $\varphi$ would result in better spectrum opportunities. However, at the cost of more interference with primary network.

Now, let consider that the sensor devices are aware of the temporal channel utilization pattern and spatial distribution of the primary transmitters of each channel. The channel accessibility status of primary user can be obtained from network service provider. Using Eq. (4) and (5) the sensor device can obtain feasible channel accessible of each channel, i.e., $\beta_{j}, j \in \mathcal{A}$, based on their mobility. Before initializing communication, the sensor device performs spectrum sensing, and is assumed to be accurate in this work. Because the channel availability is independent irrespective of scenario before the spectrum sensing and follows exponential distribution which is memoryless. The feasible channel accessible is set to zero, if channel is not accessible due to presence of primary user. We consider that the data of each sensor device is 
identical and they all move with constant speed and direction with in the sensor network and they all can communicate with each other. For accessing licensed spectrum, sensor device opportunistically accesses the channel with different feasible channel accessible parameters in a distributed nature. To evaluate the performance of distributed sensing, this work employs game theory. Each sensor device chooses a channel and tries to maximize its utility. Further, existence and adaptiveness of Nash equilibrium is presented considering random and slotted MAC and present a hybrid distributed spectrum access algorithm for CR-WSN.

\subsection{Spectrum Admittance Model using Game Theory}

The spectrum admittance problem can be modelled as a channel overloading game which is composed of set of resources/assets and actors and the cost of each actors (by choosing one assets) is proportional to the quantum of other actors choosing same resources [21]. In this work, the channel overloading game is defined as $\gamma=\left\{\mathcal{O}, \mathcal{D},\left\{T_{k}\right\}_{k \in \mathcal{O}},\left\{V_{k}\right\}_{k \in \mathcal{O}}\right\}$, where $\mathcal{O}$ is the determinate set of actors (or sensor device) in a particular group or cluster which is obtained as follows:

$$
\mathcal{O}=\{1, \ldots, O\}
$$

Ois associated to sensor density which is represented as $\alpha_{w}, \mathcal{D}$ depicts set of accessible channels (. i.e., channel sensed are considered to be idle) which is obtained as.

$$
\mathcal{D}=\{1, \ldots, D\}
$$

Where $\mathcal{D} \subseteq \mathcal{A}, T_{k}$ is the set of fine-grained policies related to the sensor device $k$, and $V_{k}$ is the utility parameter of sensor device $k$. Sensor device that take part in competition are conscious of feasible channel accessible of all channels $\left(\beta_{j}\right)$ and the amount of sensor device in the game $(O)$. The data rate of each channel is same and each sensor device is fitted with on cognitive radio, and utmost it can access only one channel at each instance, therefore, $T_{k}=\mathcal{D}$ for all $k \in \mathcal{O}$. Considering this scenario, the utility of sensor device $k$ by selecting channel $j$ is represented by $V_{k}^{j}$. An important thing to be noted here that $V_{k}^{j}$ is a parameter of both $t_{-k}$ and $t_{k}$, which are the policies chosen by sensor device $k$ and all of its competitors, respectively. In this competition, the utility $V_{k}^{j}$ is described as the mean cumulated channel resources is reengaged by primary transmitting users and is obtained as.

$$
V_{k}^{j}=\beta_{j} s\left(o_{j}\right)
$$

Where $o_{j}$ is the total number of sensor devices that concurrently select channel $j$ that includes sensor device $k$. The resource assignment parameter $s\left(o_{j}\right)$ depicts the portion of channel $j$ retrieved by each of the $o_{j}$ sensor device. For a random sensor device, $\beta_{j}$ is used to evaluate the mean time period in which channel $j$ is accessible. Therefore, $V_{k}^{j}=\beta_{j} s\left(o_{j}\right)$ depicts the mean cumulated quantum of channel asset that sensor device $k$ can get before it must terminate transmission or communication due to arrival/presence of active primary transmitting users. The channel with best $\beta_{j}$ is desired. Since, it can aid in reducing erratic channel switching and spectrum sensing and $s($.$) Depends on type of MAC we adopt. However, s($.$) should satisfy$ the underlying conditions.

$s(1)=1$. A device/contender can obtain all the resources of a particular channel if it is the only contender that exist or select that channel. $s(o)$ is a reducing function of $o$, Describe $g(o)=o s(o) . g(o)$ reduces with respect to $o$, thus $g^{\prime}(o)<0$ and $g^{\prime \prime}(o)>0 . o_{j} s\left(o_{j}\right) \leq 1$. Resource underutilization may occur when multiple contenders share the channel due to collision or contention.

Each sensor device tries to maximize its utilities because sensor devices are selfish and sensible. To examine this competition, we aim on the Nash Equilibrium. To begin with, we will examine the existence, condition and adaptive ratio of fine-grained Nash Equilibrium using slotted and random MAC, respectively. Further, we will define a spectrum access model for attaining fine-grained Nash Equilibrium with an ideal adaptive ratio.

\subsection{Nash Equilibrium in Channel Access Competition}

The Nash equilibrium states that, in the equilibrium, every contender can choose a utilitymaximizing policy, given the policies of other contenders. Here we consider a policy objective for the actors $T^{*}=\left(t_{1}^{*}, t_{2}^{*}, t_{3}^{*}, \ldots, T_{O}^{*}\right)$ is an Nash equilibrium if it satisfies the following condition. 


$$
\mathrm{V}_{\mathrm{k}}\left(\mathrm{t}_{\mathrm{k}}^{*}, \mathrm{t}_{-\mathrm{k}}^{*}\right) \geq \mathrm{V}_{\mathrm{k}}\left(\mathrm{t}_{\mathrm{k}}^{\prime}, \mathrm{t}_{-\mathrm{k}}^{*}\right) \forall \mathrm{k} \in \mathcal{O}, \mathrm{t}_{\mathrm{k}}^{\prime} \in \mathrm{T}_{\mathrm{k}}
$$

(9) Depicts that no contender can rise its utility parameter by itself by altering its own policy, given the policies of other contenders. If the policy objectives $T$ in (9) is deterministic, then it is known as a finegrained Nash equilibrium. Therefore, this work interchangeably uses the term Nash equilibrium and finegrained Nash equilibrium.

In a spectrum access competition $\gamma$, if no sensor device can enhance its utility by changing to another channel alone for a given policy objective, the policy objective is considered to be a fine-grained Nash equilibrium. Therefore, policy objectives of all sensor device that select each channel is obtained as follows:

$$
T=\left(t_{1}, t_{2}, t_{3}, \ldots, T_{O}\right)
$$

Similarly, the congestion/ channel overloading vector, which describes the number of sensor device that select each channel, with respect to the policy objective $T$ is obtained as follows:

$$
o(T)=\left(o_{1}, o_{2}, o_{3}, \ldots, o_{\mathcal{D}}\right)
$$

Therefore, the spectrum access completion $\gamma$ has fine-grained Nash equilibriums if it satisfies the following condition for each actor $k \in \mathcal{O}$.

$$
\beta_{t_{k}} s\left(o_{t_{k}}\right) \geq \beta_{a} s\left(o_{a}+1\right), \forall a \in \mathcal{D} a \neq t_{k}
$$

Normally it is seen that there exist multiple policy objectives with respect to channel overloading vector $O^{*}$ is fine-grained Nash equilibrium, as a result all policy objectives with respect to $O^{*}$ are fine-grained Nash equilibriums based on (12). Let us consider a set of fine-grained Nash equilibriums $\mathbb{N}(o)$ with respect to channel overloading vector $o$. The Nash equilibriums in $\mathbb{N}(o)$ may obtain varied utilities for each actor. However, they attain the same cumulated utilities, which can be described as summation of the utilities of all sensor device which is obtained as follows:

$$
V_{\text {cum }, O}=\sum_{j=1}^{D} \gamma_{j} o_{j} s\left(o_{j}\right)=\sum_{j=1}^{D} \gamma_{j} g\left(o_{j}\right)
$$

where $o=\left(o_{1}, o_{2}, o_{3}, \ldots, o_{D}\right)$. In next section, we evaluate spectrum access completion of Nash equilibrium and present adaptive random MAC design for CRWSN.

\subsection{Adaptive Spectrum or Channel Access Design for CR-WSN}

The ideal method of sharing the resources among multiple contenders is to make each contender to access the channel ideally in probable manner, which is considered as an Adaptive random MAC [22-23]. Each user initializes its back-off time, arbitrarily selected from a static window. If one sensor device establishes that its back-offs expires and channel is idle, it can occupy the channel of entire time slot, while another sensor device must be quiet. In random MAC, the resource assignment strategy $s(o)=1 / o$. Therefore, the utility function can be represented as follows:

$$
V_{k\{r n d\}}^{j}=\frac{\beta_{j}}{o_{j}}
$$

where $g_{r n d}(o)=1$. Fine-grained Nash equilibrium for random MAC can be obtained considering following condition. Let consider a spectrum access completion $\gamma$ for random MAC, if a channel overload vector $o=$ $\left(o_{1}, o_{2}, o_{3}, \ldots, o_{D}\right)$ attains Nash equilibrium set $(o)$, the corresponding state must be satisfied.

$$
\left\{\begin{array}{c}
o_{j}=\left\lceil\frac{\beta_{j} O-\sum_{a \neq j, a \in D} \beta_{a}}{\sum_{a \in D} \beta_{a}}\right\rceil+X_{0} j=1,2,3, \ldots, D \\
\sum_{j=1}^{D} o_{j}=O
\end{array}\right.
$$

where $X_{0} \in\left\{0,1,2,3, \ldots,\left\lceil\beta_{j}|O|+\beta_{j}(|\mathcal{D}|-1) / \sum_{a \in D} \beta_{a}\right\rceil-\left\lceil\beta_{j}|O|-\sum_{a \neq j, a \in D} \beta_{a} / \sum_{a \in D} \beta_{a}\right\rceil-1\right\}$.

In general, a Nash equilibrium does not attain global optimal solution due to egoistic behavior contender. The adaptiveness performance of a Nash equilibrium is examined to compute the resource utilization, which is here referred as the total utility of all contenders considering Nash equilibrium. And 
considering (13), in the spectrum access competition $\gamma$, the adaptiveness performance of a fine-grained Nash equilibrium is represented as follows:

$$
\mathbb{A}_{T}=\sum_{k=1}^{O} V_{k}^{j}=\sum_{k=1}^{O} \beta_{t_{k}} s\left(o_{t_{k}}\right)=\sum_{j=1}^{O} \beta_{j} o_{j} s\left(o_{j}\right)
$$

where $T$ is a solution profile that is a fine-grained Nash equilibrium. Similarly, the social optimal solution is described as a peak cumulated utility of all contenders among all probable policy profiles. The social optimality of certain competition is fixed [22-23] i.e., the social optimal solution in $\gamma$ is obtained as follows:

$$
\mathbb{O}_{\gamma}=\left\{\begin{array}{c}
\sum_{j=1}^{O} \beta_{j}, \text { if } O \leq D \\
\left.\sum_{j=1}^{D-1} \beta_{j}+\sum_{j=D}^{O} \beta_{D} s()-D+1\right) \text {, if } O>D
\end{array}\right.
$$

where $\beta_{j}$ is ordered in such way that $\beta_{1} \geq \beta_{2} \geq \beta_{3} \geq \cdots \geq \beta_{D}$. Therefore, to compute the adaptiveness performance of Nash equilibrium, we describe the adaptiveness ratio of a Nash equilibrium as the ratio among the adaptiveness performance and social optimal solution as follows:

$$
\mathbb{A} \mathbb{R}_{S}=\mathbb{A}_{T} / \mathbb{O}_{\gamma}
$$

Different Nash equilibrium set may attain different adaptive ratios. The adaptiveness ratio of finegrained Nash equilibrium using random MAC is discussed. In Random MAC, $g(o)=1$. Among multiple Nash equilibrium sets with different channel overhead vectors, we can conclude that firstly, A fine-grained Nash equilibrium in which each channel is selected by at most one sensor device has $\mathbb{A} \mathbb{R}$. For any two different Nash equilibrium sets (NashEqlb - sets) in which not all channels are selected, if:

$$
\sum_{j=1}^{O} \beta_{j} J_{j}^{1}>\sum_{j=1}^{O} \beta_{j} J_{j}^{2}
$$

Where $J_{j}^{1}$ is depicts whether channel $j$ is selected in NashEqlb - sets ${ }_{k}$, then $\mathbb{A R}_{1} \geq \mathbb{A} \mathbb{R}_{2}$. It is clear that when employing random MAC, the adaptiveness performance equals the summation of the feasible channel accessibility of entire channels that are chosen, that is, $\mathbb{A}_{T}=\sum_{j=1}^{D} \beta_{j} J_{j}^{T}$. When entire channels are selected, all resource is utilized fully, and therefore, $\mathbb{A} \mathbb{R}=1$. Else, the higher adaptive performance attains a superior $\mathbb{A} \mathbb{R}$, because the social optimal solution are static for a particular competition.

\subsection{Hybrid Spectrum Access Model}

To address the issue and attain a fine-grained Nash equilibrium with good adaptive ratio performance in a distributed nature, we present a hybrid spectrum access design which is distributed in nature as shown in Algorithm 1.

Algorithm 1: Hybrid spectrum access model

Step 1: Start

Step 2: Obtain accessible channel $\mathcal{D}$ by sensing

Step 3: Update and set (inform) the channel accessibility $\left[\beta_{1}, \beta_{2}, \beta_{3}, \ldots, \beta_{D}\right]$ decreasingly using (4) and (5) considering that the present instance is $u_{t}$.

Step 4: Each sensor device that request for communication opportunity selects an arbitrary back-off time $u_{c}$ from $\left(0, u_{c^{\uparrow}}\right)$ and initialize the back-off.

Step 5: While present instance $\leq\left(u_{t}+u_{c} \uparrow\right)$ do

Step 6: if the back-off timer of sensor device $j$ finishes then

Step 7: if random MAC then

Step 8: Choose the best response with a free channel, considering the solution that it obtains. If entire channel has been selected, then chose any best response.

Step 9: End if

Step 10: if slotted MAC then

Step 11: Choose the channel with higher adaptive performance.

Step 12: End if

Step 13: Broadcast the channel sequence ID that selects.

Step 14: End if

Step 15: End while

Hybrid spectrum access model using game theory approach for multi-channel heterogeneous... (Saroja T.V) 
Step 16: Each sensor device selects ideal channel and start communication using a particular MAC.

Step 17: End.

Each sensor device will arbitrarily choose a back-off time and initialize the back-off. When the back-off instance ends, the sensor device selects a channel to communicate based on best response to the policies of sensor device that have previously selected the channel. Then, the sensor device broadcast its choice for other sensor devices to describe their policies. Since the back-off time selection is arbitrary, therefore the proposed design is fair for each sensor device and attain good overall networks performance compared with state-of-art techniques which is experimentally proved in net section below.

\section{SIMULATION RESULT AND ANALYSIS}

This section presents performance evaluation of HSAM-CRWSN over exiting model [15, 24] in terms of throughput, successful packet transmission, and collision. The experiments are conducted on windows 10 enterprise edition, Intel Pentium I-7 class processor, 16 GB RAM with 4 GB dedicated CUDA graphic card. The HSAM-CRWSN and exiting model is implemented using Dot Net framework 4.5 and C\# programing language. Performance of HSAM-CRWSN and exiting model is evaluated considering varied network density and mobility using following simulation parameter presented in Table 1.

Table 1. Simulation Parameter Considered

\begin{tabular}{cc}
\hline Network Parameter & Value \\
\hline Network Size & $50 \mathrm{~m} * 50 \mathrm{~m}$ \\
Number of Sensor device & $20,40 \& 80$ \\
Initial energy of sensor device & 1.0 joules \\
Modulation scheme & QAM- 64 \\
Mobility of devices & $4,6,8$ cycles per frame \\
Coding rate & 0.75 \\
Bandwidth & $27 \mathrm{Mbps}$ \\
Number of Frequency Channels & 7 \\
Time slots & $8 \mu \mathrm{s}$ \\
Message information size & 27 bytes \\
MAC used & Hybrid MAC
\end{tabular}

\subsection{Throughput Performance Considering Varied Network Density}

Here experiments are conducted to evaluate the performance of HSAM-CRWSN over existing model in terms of throughput achieved per channel considering varied sensor device. The sensor nodes are varied from 20, 40 and 80 and simulation is conducted and result are plotted as shown in Figure 2. Each sensor device moves at identical and fixed speed and direction. Lastly, this work assumes that each sensor nodes can communicate with each other. Experiment outcome shows HSAM-CRWSN improves throughput performance by $14.96 \%, 18.49 \%$, and $22.22 \%$ over existing model considering 20,40 , and 80 sensor devices, respectively. An average improvement of $18.55 \%$ is achieved by HSAM-CRWSN over existing model in terms of throughput achieved per channel considering varied sensor device. The overall result shows that HSAM-CRWSN performs significantly better than exiting model considering varied sensor devices which shows adaptiveness of HSAM-CRWSN.

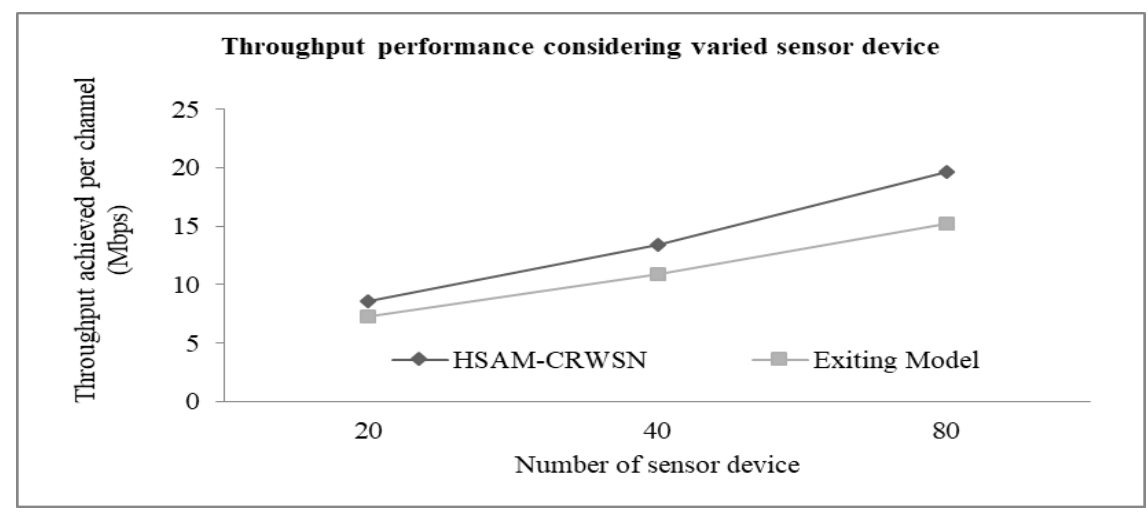

Figure 2. Throughput performance considering varied sensor device 


\subsection{Successful Packet Transmission Performance Considering Varied Network Density}

Here experiments are conducted to evaluate the performance of HSAM-CRWSN over existing model in terms of successful packet transmission considering varied sensor device. The sensor nodes are varied from 20, 40 and 80 and simulation is conducted and result are plotted as shown in Figure 3. Each sensor device moves at identical and fixed speed and direction. Lastly, this work assumes that each sensor nodes can communicate with each other. Experiment outcome shows HSAM-CRWSN improves successful packet transmission performance by $21.05 \%, 31.76 \%$, and $25.21 \%$ over existing model considering 20, 40, and 80 sensor devices, respectively. An average improvement of $26.01 \%$ is achieved by HSAM-CRWSN over existing model in terms of successful packet transmission considering varied sensor device. The overall result shows HSAM-CRWSN performs significantly better than exiting model considering varied sensor device which shows adaptiveness of HSAM-CRWSN.

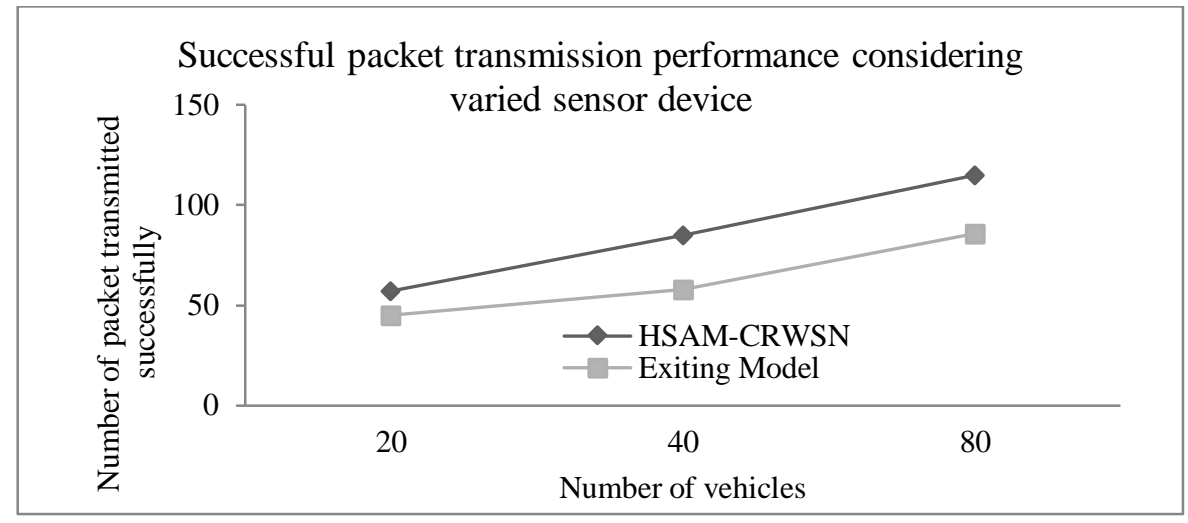

Figure 3. Successful packet transmission performance considering varied sensor device

\subsection{Packet Collision Performance Considering Varied Network Density}

Here experiments are conducted to evaluate the performance of HSAM-CRWSN over existing model in terms of packet collision considering varied sensor device. The sensor nodes are varied from 20, 40 and 80 and simulation is conducted and result are plotted as shown in Figure 4. Each sensor device moves at identical and fixed speed and direction. Lastly, this work assumes that each sensor nodes can communicate with each other. Experiment outcome shows HSAM-CRWSN reduces packet collision by $34.78 \%, 34.78 \%$, and $18.63 \%$ over existing model considering 20, 40, and 80 sensor devices, respectively. An average reduction of $29.39 \%$ is achieved by HSAM-CRWSN over existing model in terms of packet collision considering varied sensor device. The overall result shows HSAM-CRWSN performs significantly better than exiting model considering varied sensor device which shows adaptiveness of HSAM-CRWSN.

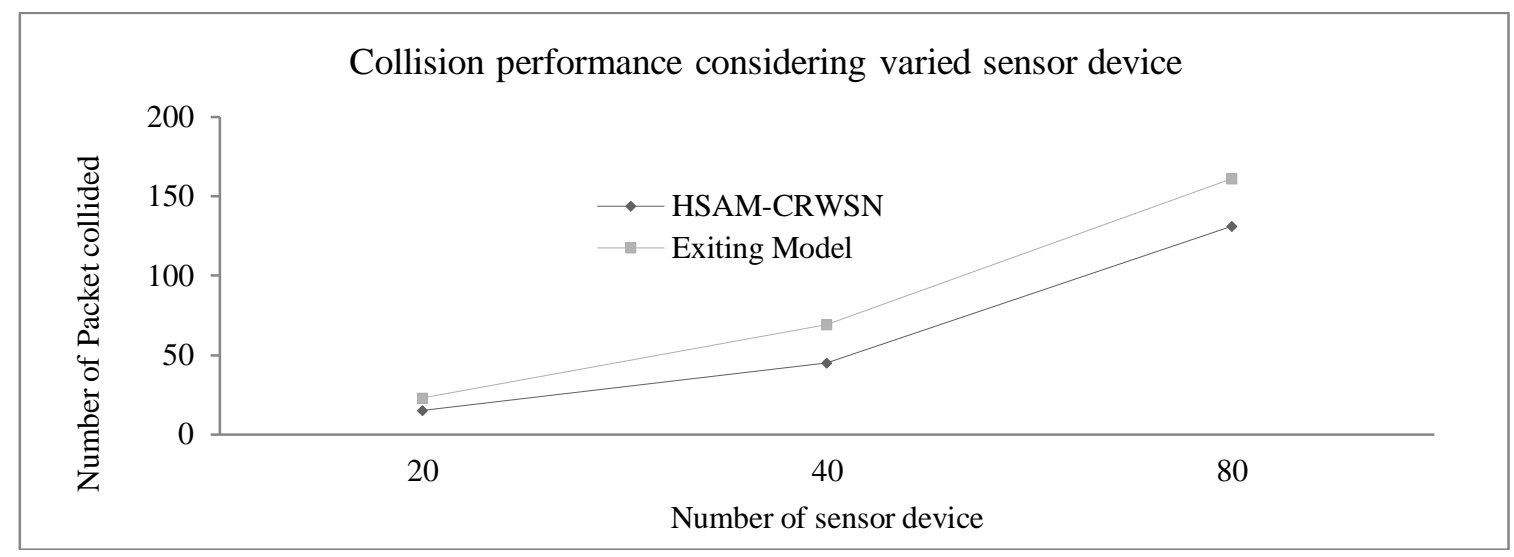

Figure 4. Packet collision performance considering varied sensor devices 


\subsection{Performance Evaluation Considering Varied Mobility Speed of Sensor Device}

Here experiments are conducted to evaluate the performance of HSAM-CRWSN over existing model in terms of throughput and packet collision considering varied sensor mobility speed. The network density size is fixed 60 sensor devices. The sensor nodes speed is varied from are varied from 4 (maximum speed), 6, and 8 (minimum speed) cycle per frame and simulation is conducted and result are plotted for throughput and packet collision as shown in Figures 5 and 6. Experiment outcome shows HSAM-CRWSN reduces packet collision by $35.15 \%, 50.0 \%$, and $60.34 \%$ and improves throughput by $30.72 \%, 30.15 \%$, and $20.78 \%$ over existing model considering 4, 6, and 8 cycles per frame, respectively. An average reduction of $48.5 \%$ is achieved by HSAM-CRWSN over existing model in terms of packet collision considering varied mobility speed. An average improvement of $27.22 \%$ is achieved by HSAM-CRWSN over existing model in terms of throughput considering varied mobility speed. The overall result shows HSAM-CRWSN performs significantly better than exiting model considering varied mobility speed of sensor device which shows adaptiveness of HSAM-CRWSN.

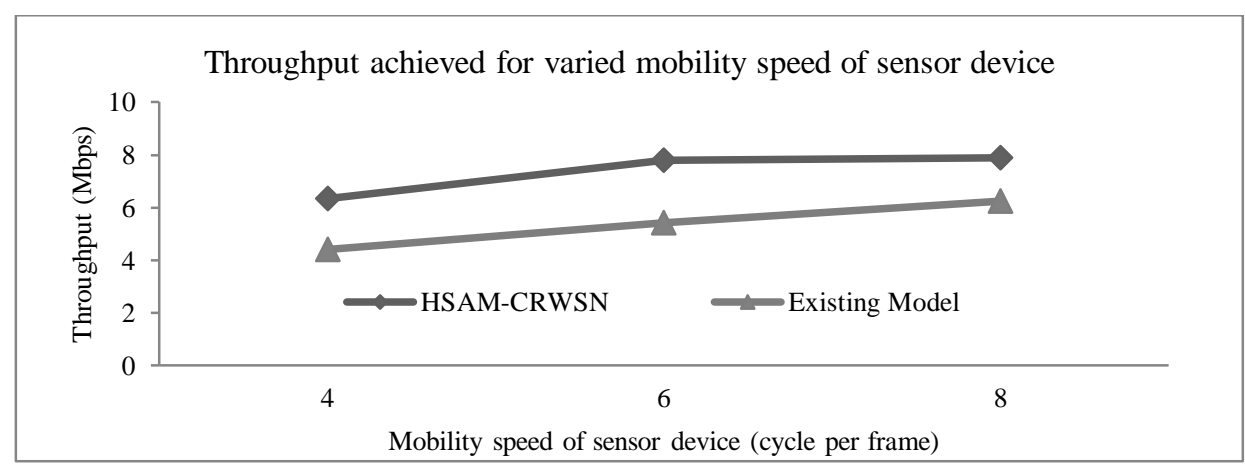

Figure 5. Throughput performance considering varied sensor devices

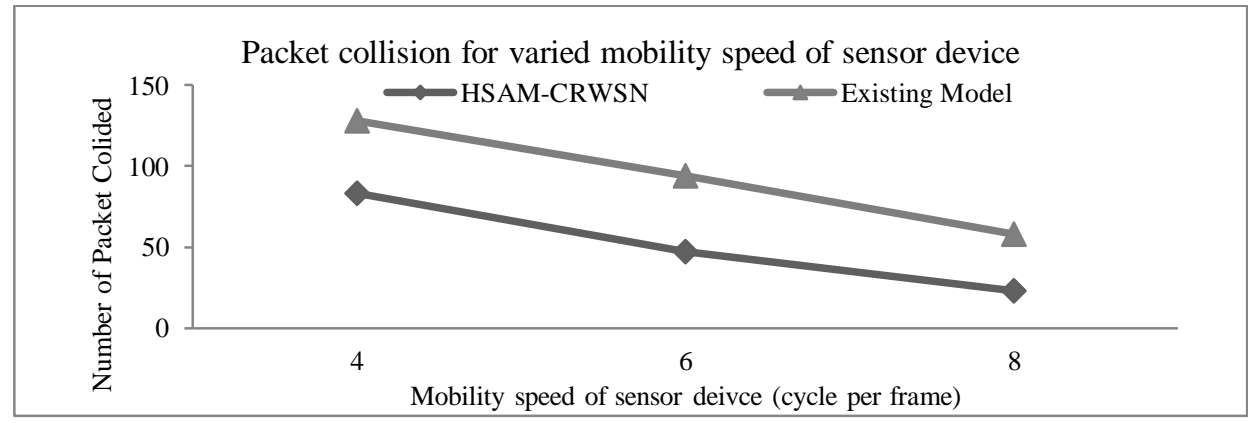

Figure 6. Packet collision performance considering varied sensor devices

\subsection{Result and Discussion}

The overall result attained shows the HSAM-CRWSN significant performance improvement over state of art technique $[15,24]$ in terms of throughput, successful packet transmission and collision. Further, this work evaluates energy efficiency of HSAM-CRWSN. The parameter and energy consumption model used to evaluate energy efficiency of HSAM-CRWSN is obtained from [7] and the energy efficiency $\mathbb{E}$ of secondary user is computed as follows $[18,25]$.

$$
\mathbb{E}=\frac{\mathcal{T}}{\mathcal{S}}
$$

where $\mathcal{T}$ is the amount of effective packet transmitted and $\mathcal{S}$ is the expected energy consumed. Here we compare HSAM-CRWSN with [18] only. Since, as per our knowledge it is only joint spectrum access model for multi-channel CR-WSN. Experiment outcome shows HSAM-CRWSN attains an average energy efficiency of $21.34 \%, 26.01 \%$, and $31.04 \%$ over Two-Layer Game [18] considering varied sensor device, sensor mobility speed, and frequency channel. The outcome attained is due to accurate channel available 
likelihood estimation model and existence of fine-grained Nash equilibrium for hybrid spectrum access model that reduces interference among users and the same time allocate resource fairly to user. Thus, reducing collision, increasing packet transmission and aid in achieving higher throughput. As a result, utilize spectrum efficiently.

\section{CONCLUSION}

In CR network, the spectrum is generally not occupied by primary licensed users all the time. Therefore, utilizing this unoccupied spectrum plays a critical role in providing efficient resources to end clients. For providing efficient services the sensor (IoT) device can exploit these unused slots for data transmission. However, designing spectral, energy efficient and fair spectrum access design for IoT in CRWSN is challenging especially for heterogeneous mobile CR-WSN. To meet the research challenges, at first a hybrid spectrum access model for multi-channel heterogeneous mobile CR-WSN is presented. For addressing collision due to interference this paper proposes an accurate channel accessible likelihood estimation using continuous-time Markov chain model. Finally, a game theory model for HSAM for existence of fine-grained Nash equilibrium is presented. Experiment is conducted to evaluate the performance of proposed HSAM-CRWSN over state-of-art technique in terms of energy efficiency, throughput, successful packet transmission and collision considering varied network density, Mobility speed and frequency channels. The outcome shows significant performance improvement. An average throughput performance improvement of $18.55 \%$ and $27.22 \%$ is achieved by proposed HSAM-CRWSN over existing model considering varied network density and speed. An average successful packet transmission performance improvement of $26.01 \%$ is achieved by proposed HSAM-CRWSN over existing model considering varied network density. An average collision reduction of $28.39 \%$ and $48.5 \%$ is achieved by proposed HSAM-CRWSN over existing model considering varied network density and speed. An average energy efficiency performance improvement of $21.34 \%, 26.01 \%$, and $31.04 \%$ is achieved by proposed HSAM-CRWSN over existing over Two-Layer Game model considering varied network density, speed, and frequency channel. The overall result shows HSAM-CRWSN attains significant performance improvement over state-of-art technique in terms of energy efficiency, throughput, successful packet transmission and packet collision. HSAM-CRWSN brings a good tradeoff between energy efficiency and performance requirement of future real-time applications of CR-WSN. The HSAM-CRWSN improves the overall spectrum efficiency of CR-WSN. The future work would consider performance evaluation considering varied network parameter and also evaluating access fairness performance of HSAM-CRWSN. Further, consider optimizing HSAM for attaining better performance.

\section{REFERENCES}

[1] Lin Y, Yang J, Lv Z, et al. “A self-assessment stereo capture model applicable to the internet of things". Sensors, 15(8): 20925-20944, 2015.

[2] Yang J, He S, Lin Y, et al. "Multimedia cloud transmission and storage system based on internet of things". Multimedia Tools and Applications, 2015: 1-16, 2015.

[3] Naranjo, Paola G. Vinueza, et al. "P-SEP: a prolong stable election routing algorithm for energy-limited heterogeneous fog-supported wireless sensor networks." The Journal of Supercomputing, (2016): 1-23.

[4] N. Li, M. Xiao and L. K. Rasmussen, "Spectrum Sharing with Network Coding for Multiple Cognitive Users," in IEEE Internet of Things Journal. doi: 10.1109/JIOT.2017.2728626.

[5] Aijaz and A. H. Aghvami, "Cognitive machine-to-machine communications for Internet-of-Things: A protocol stack perspective," IEEE Internet Things J., vol. 2, no. 2, pp. 103-112, Apr. 2015.

[6] T. M. Chiwewe and G. P. Hancke, "Fast Convergence Cooperative Dynamic Spectrum Access for Cognitive Radio Networks," in IEEE Transactions on Industrial Informatics. doi: 10.1109/TII.2017.2783973.

[7] S. Aslam, W. Ejaz and M. Ibnkahla, "Energy and Spectral Efficient Cognitive Radio Sensor Networks for Internet of Things," in IEEE Internet of Things Journal. doi: 10.1109/JIOT.2018.2837354.

[8] W. Ejaz, G. A. Shah, H. S. Kim et al., "Energy and throughput efficient cooperative spectrum sensing in cognitive radio sensor networks," Transactions on Emerging Telecommunications Technologies, vol. 26, no. 7, pp. 10191030, Jul. 2015.

[9] Maghsudi S, Stanczak S. "Hybrid Centralized-Distributed Resource Allocation for Device-to-Device Communication Underlaying Cellular Networks". IEEE Transactions on Vehicular Technology, 2016.

[10] Yuhua Xu; Jinlong Wang; Qihui Wu; Anpalagan, A.; Yu-Dong Yao. "Opportunistic Spectrum Access in Unknown Dynamic Environment: A Game-Theoretic Stochastic Learning Solution,” IEEE Trans. Wireless Communications, pp. 1380-1391, 2012.

[11] Yuhua Xu; Jinlong Wang; Qihui Wu; Anpalagan, A.; Yu-Dong Yao. “Opportunistic Spectrum Access in Unknown Dynamic Environment: A Game-Theoretic Stochastic Learning Solution,” IEEE Trans. Wireless Communications, pp. 1380-1391, 2012. 
[12] Anandkumar, N. Michael, A. Tang, and A. Swami, "Distributed algorithms for learning and cognitive medium access with logarithmic regret," IEEE J. Sel. Areas Commun. on Advances in Cognitive Radio Networking for Communications, vol. 29, pp. 731-745, Mar. 2011.

[13] S. Vakili, K. Liu, and Q. Zhao, "Deterministic sequencing of exploration and exploitation for multi-armed bandit problems," IEEE J. Sel. Topics Signal Process., vol. 59, no. 3, pp. 1902-1916, Oct. 2013.

[14] Y. Gai and B. Krishnamachari, "Decentralized online learning algorithms for opportunistic spectrum access," in Proc. IEEE Global Commun. Conf. (GLOBECOM), Dec. 2011, pp. 1-6.

[15] Y. Xu, Q. Wu, J. Wang, L. Shen and A. Anpalagan, "Robust Multiuser Sequential Channel Sensing and Access in Dynamic Cognitive Radio Networks: Potential Games and Stochastic Learning," in IEEE Transactions on Vehicular Technology, vol. 64, no. 8, pp. 3594-3607, Aug. 2015.

[16] F. Zhou, N. C. Beaulieu, J. Cheng, Z. Chu and Y. Wang, "Robust Max-Min Fairness Resource Allocation in Sensing-Based Wideband Cognitive Radio With SWIPT: Imperfect Channel Sensing," in IEEE Systems Journal. doi: 10.1109/JSYST.2017.2698502.

[17] H. Jang, S. Y. Yun, J. Shin and Y. Yi, "Game Theoretic Perspective of Optimal CSMA," in IEEE Transactions on Wireless Communications, vol. 17, no. 1, pp. 194-209, Jan. 2018. doi: 10.1109/TWC.2017.2764081

[18] Y. Lu and A. Duel-Hallen, "A Sensing Contribution-Based Two-Layer Game for Channel Selection and Spectrum Access in Cognitive Radio Ad-hoc Networks," in IEEE Transactions on Wireless Communications, vol. 17, no. 6, pp. 3631-3640, June 2018.

[19] Saroja T.V, Lata L. Ragha, Satyendra Kumar Sharma, “A Dynamic Spectrum Access Optimization Model for Cognitive Radio Wireless Sensor Network" 2017.

[20] T. V. Saroja and L. L. Ragha, "A dynamic spectrum access model for cognitive radio wireless sensor network," 2017 4th International Conference on Electronics and Communication Systems (ICECS), Coimbatore, pp. 7-11, 2017.

[21] D. Chakrabarty, A. Mehta, V. Nagarajan, and V. Vazirani. Fairness and optimality in congestion games. In EC'05, 2005.

[22] E. Koutsoupias and C. Papadimitriou. "Worst-case equilibria". In Proceedings of the 16th Annual Symposium on Theoretical Aspects of Computer Science, pages 404-413, 1999.

[23] T. Roughgarden and E. Tardos. How bad is selfish routing? Journal of the ACM, 49(2):236-259, 2002.

[24] P. Spachos and D. Hatzinakos, "Real-Time Indoor Carbon Dioxide Monitoring Through Cognitive Wireless Sensor Networks," in IEEE Sensors Journal, vol. 16, no. 2, pp. 506-514, Jan.15, 2016.

[25] X. Hao, M. H. Cheung, V. W. S. Wong, and V. C. M. Leung, "Hedonic coalition formation game for cooperative spectrum sensing and channel access in cognitive radio networks, "IEEE Trans. Wireless Commun., vol. 11, no. 11, pp. 3968-3979, 2012. 